\title{
The implementation of multicast with SIP in distance education system
}

\author{
${ }^{\mathrm{a}}$ LI Xiao-hua, ${ }^{\mathrm{b}} \mathrm{LIU}$ Shu-xian \\ ${ }^{a}$ Computer Science InstitutionXinjiang Normal University Urumqi Xinjiang, China \\ ${ }^{b}$ School of Information Science and Engineering Xinjiang University Urumqi Xinjiang , China
}

\begin{abstract}
This paper introduce the difference between single-cast and multicast briefly, and analysis the method of multicast and their shortcomings in the traditional network system and in H.323 system. The article discusses how to implement multicast in SIP system particularly, and separately describes route of single-cast and multicast in SIP system in detail.
\end{abstract}

Index Terms: Session Initiation Protocol (SIP); multicast; distance education system

(C) 2012 Published by MECS Publisher. Selection and/or peer review under responsibility of the International Conference on E-Business System and Education Technology

\section{Introduction}

\subsection{Origin of SIP}

SIP is Session Initiation Protocol, which is a signaling protocol used to implement instant message based on IP network[1].

SIP use the textual encoded mode which is the best feature of SIP when comparing with other VoIP and the existing standard in video telecommunication domain[2].

\subsection{Characteristics of SIP}

First, expansibility of SIP

SIP has a powerful extension mechanism. The expansion is happened mainly in the aspect of messages. Therefore, this message based approaching made the extension work quite easy[2].

Second, opening generating environment for the business

SIP internet services are mainly done by the proxy server. The appearance of a business is to design business logic to control the certain message flow which could be carry out by any kinds of general-purposed programming languages.

Third, mobility

Corresponding author:

E-mail address: ${ }^{\mathrm{a}}$ lxhxjnu@126.com, blsx623@163.com 
The dynamically register system provides the convenience of mobility to clients. SIP made the seamless fusion of landline services and wireless services possible. SIP had been chosen to be the signaling in the multimedia domain for the $3 \mathrm{G}$ mobile communication. It is used for the realization of the mobile voice and multimedia communication based on IP.

\subsection{SIP System}

SIP system has four main components:

SIP UA (User Agent) : It is the end-user's equipment. Users employ UAC (User Agent Client) to send messages. UAS (User Agent Server) responds to those messages[3].

SIP RS (Registrar) : It is a data base with all the clients' proxy location in the domain. In the process of SIP communication the server will search the IP addresses and other related information of participates and send them to the SIP proxy server[3].

SIP PS (Proxy Server) : It is used to accept the conversation request from SIP UA and to inquire SIP RS to get the address information of receiver UA[3].

SIP RDS (Redirect Server) : It allows SIP proxy server redirect the SIP invitation information to the external domain[3].

\section{Unicast and Multicast}

\subsection{Unicast}

Internet is designed to transfer datagram between two hosts. One host has destination IP address and the router will send those datagram to the destination. Generally speaking, one IP address marks one network-interface of a host. Once a host acts as sender and the other host is receiver, this mechanism enables any two hosts exchange data within Internet. Receiver could send back those data to the sender by recording of the sender's IP address. This route, namely using an IP address mark one host interface, is called unicast.

\subsection{Multicast}

Obviously, for the users with large-scale needs, unicast system cannot extend well. A little bit increase of participation of the host would lead dramatically increase for the network traffic which will limit the host's processing ability. Multicast router provides large-scale groups multipoint communication to solve the problem. Those routes cooperate to transfer IP groups. As for groups with different destination address, they are specified as the same multicast address. One multicast address is on behalf of a group of hosts, the hosts in the same group are willing to receive the IP packet which is to be sent[4].

\section{Multicast in the traditional IP network}

While multicasting in the traditional IP network, not only the hosts of a group should set the same multicast address, all the routes along the way of message transfer must support IGMP (Internet Group management Protocol).In the IPv4[RFC791], addresses between 224.0.0.0 239.255.255.255 are reserved for multicast use. It is easy to set the terminal address of the multicast address in the same group, but it is difficult to fulfill the requirement of making all the routers along the way of transferring to support IGMP(Internet Group Management Protocol) [5].

\section{Using SIP to multicast in the distance learning system}

\section{1 Unicast and multicast within SIP}


Unicast and multicast within SIP refer to use several unicast to achieve multicasting in SIP system.

SIP is designed as distributed call system which has distributed multicasting function. This multicasting function is not only facilitated conference control but also simplified the user location and group invitations. Besides, it saves bandwidth.

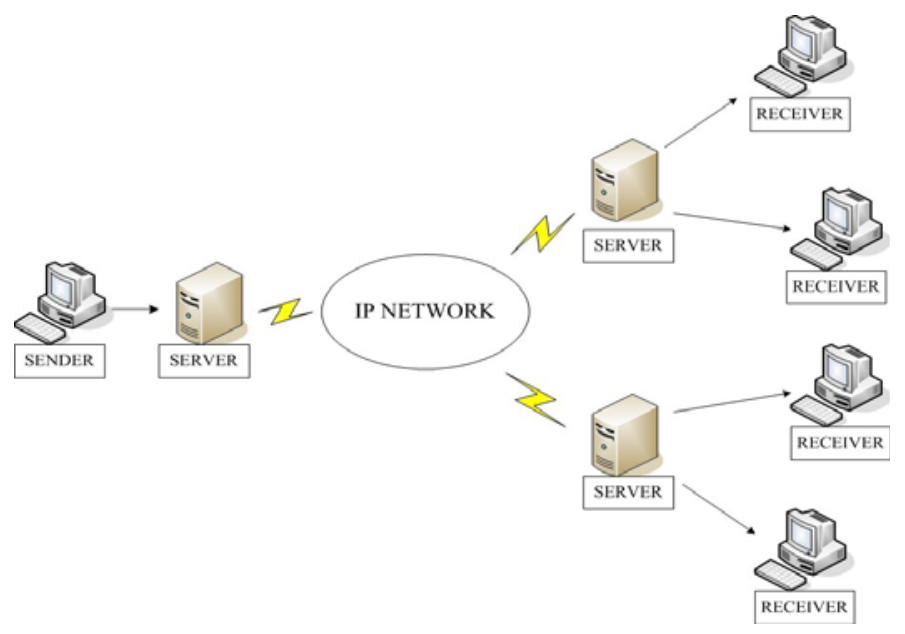

Figure 1. Multicast in unicast way

In SIP system, the user agent client (UAC) send INVITE request with a multicast message to invite the corresponding clients to participate, choosing the route through the protocol header's via session, protocol header Record-Route records route which enable multicast messages to reach each domain server. Then domain server distributes messages to the receiver through the agent distributed approach. Both core server and server are calling stateful proxy, they record the path from the beginning of the INVITE request to the end of the BYE request. Therefore, they are always in the pathway which was chosen to transfer SIP messages by the end-user. Unicast messages and multicast messages are send by the system through different processes and paths, the following two charts make a detailed comparison.

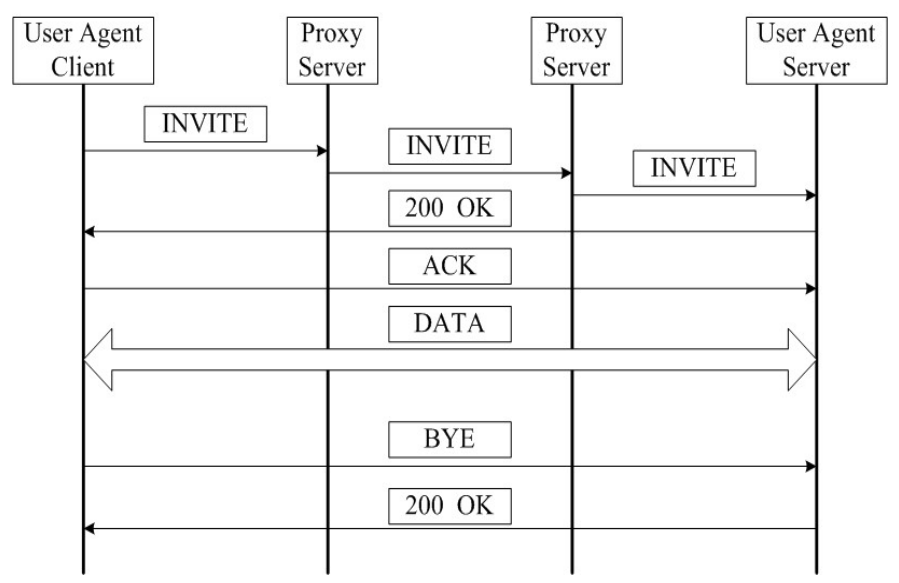

Figure 2. Pathway in unicast mode

In unicast mode, the proxy server is in the state of no reservation, the user agent clients (UAC) finally locate

The project of Education Sciences Planning of the Xinjiang Uygur Autonomous Region of China, "Research of software development and application on distance learning system” (ID:070706) 
the user agent server(UAS) through a proxy server (single or multiple) after which messages delivered directly between the two ends and then no proxy server supports are needed.

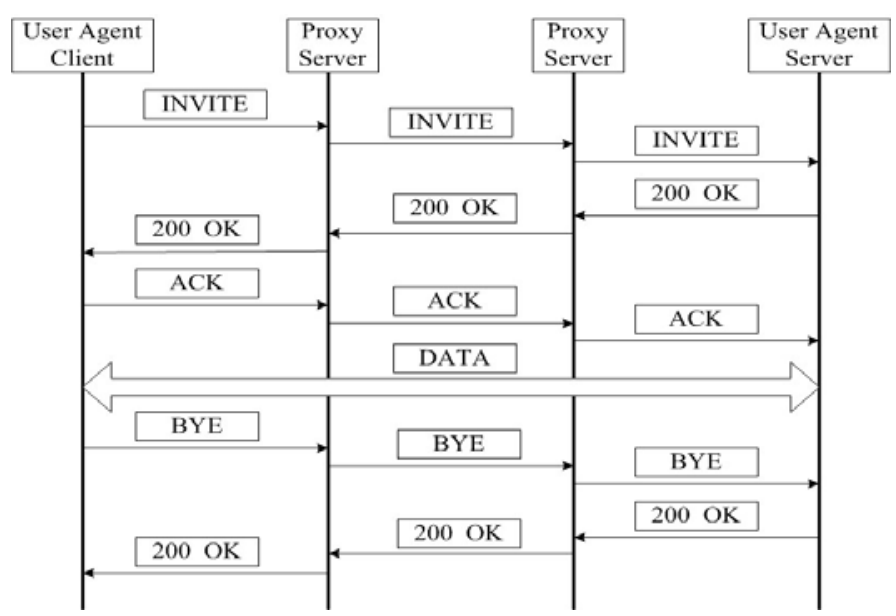

Figure 3. Pathway in multicast mode

In multicast mode, the proxy server(PS) is in the state of no reservation the user agent clients (UAC) finally locate the user agent server (UAS)through a proxy server (single or multiple) after which messages still need to be transferred through proxy server till the session termination request (BYE) transmission is completed. Although this transmission method burdened the proxy server, it guaranteed the realization of multicast capabilities.

\section{2 Broadcast Multicast in SIP}

In the distance education system, the receiver is often in a rather special network environment, like, all (or most) receivers are belong to the same LAN. Namely, all the students are in the same classroom and receiving class information at the same time. This special case often appears in the existing distance education system.

Therefore, we can use technology of LAN broadcast to achieve multicast, that is to say the server should broadcast program information in the whole LAN (or a subnet, a VLAN). This has many advantages like greatly reducing the burden on the server, the server does not need to retain call state, the transmission of the signaling link is also greatly reduced. As the local area network is highly reliable, even if signaling reduced it will not affect the quality of transmission.

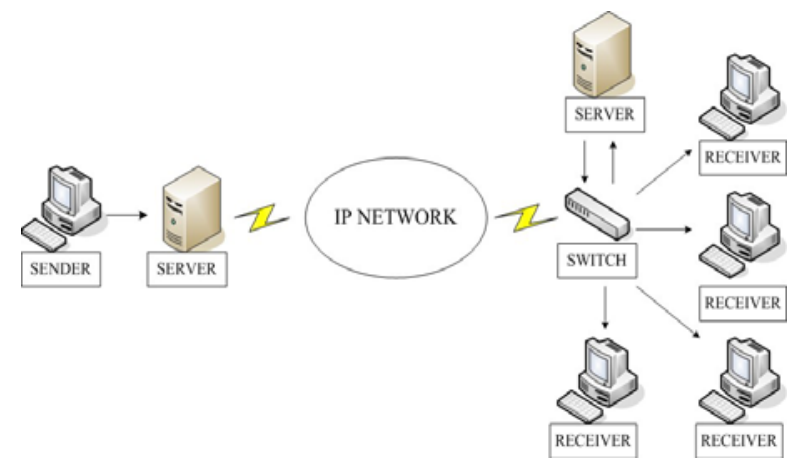

Figure 4. Broadcast way multicast in SIP system 
Multicast by broadcast is much more difficult than by unicast since the protocol stack is more complex. After the receiver's registration to the server, registration server(RS) will analyze if the receiver's registered IP belong to the same local area network, if so, it will record their IP and convert them into the local area network's broadcast address. Then, the real-time multimedia information will be sent by server in the form of the broadcast address and broadcasted by the switch.

\section{3 Mixed multicast in SIP}

Hybrid Multicast mixed the first two multicast ways. In the distance education system, the cases, like a number of Internet users log on a portable computer system to view information or to participate in classroom learning, cannot be ruled out. Obviously, the broadcast multicast approach does not apply to such users, on the premise of fulfilling the broadcast users unicast mode could be used to provide services to those cuntomers.

\section{Conclusion}

This article describes the three ways of SIP protocol multicast transmissions in the distance education system, explains in detail about how to construct a multicast message header when a multicast user agent client need to achieve unicast in the SIP system and how to record information to enable themselves in the transmission path all the time when server which is in a reservation state processing. Besides, explaination of the application of multicast by broadcast in the SIP system is presented.

SIP protocol is selected as the next generation network (NGN) framework agreement, multicast based on SIP protocol will be widely used in multi-party video conferencing, instant messaging and distance learning system. This article gives a general design to the realization of multicast using SIP in the distance education system. In specific applications amend should be made accordingly based on the number of users, media handling, application network environment constraints.

\section{References}

[1]. Zhangzhijiang,Zhangyunyong and Liuyunjie,“SIP, Protocol and Applications,”Publishing House of Electronics Industry,Beijing,2005 (in chinese).

[2]. Gonzalo Camarillo,“SIP Demystified,”Post \& Telecom Press,Beijing,2003.

[3]. CGIC online ,http://www.boutell.com/cgic/.

[4]. Siduanfeng,Hanxinhui and Longqing,“Technology core and developing in SIP,” Journal of Software,sevevteenth periodical,vol.2,pp.239-250.

[5]. Rosenberg, H. Schulzrinne and G. Camarillo,Request for Comments: 3261 SIP: Session Initiation Protocol [S],2002. 